\title{
Fuente de alimentación de ejemplares de Triatoma sordida en un área con alto riesgo de domiciliación en el Chaco Paraguayo
}

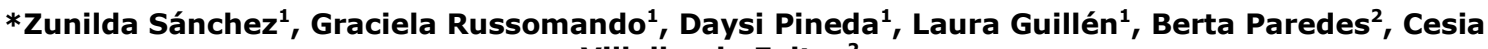 \\ Villalba de Feltes ${ }^{2}$ \\ ${ }^{1}$ Departamento de Biología Molecular y Biotecnología, Instituto de Investigaciones en Ciencias de la Salud, \\ Universidad Nacional de Asunción. Paraguay \\ ${ }^{2}$ SENEPA, Ministerio de Salud Pública y Bienestar Social. Asunción, Paraguay
}

\begin{abstract}
Cómo referenciar este artículo/
\end{abstract}
How to reference this article:
Sánchez Z, Russomando G, Pineda D, Guillén L, Paredes B, Villalba de Feltes C. Fuente de alimentación de ejemplares de Triatoma sordida en un área con alto riesgo de domiciliación en el Chaco Paraguayo. Mem. Inst. Investig. Cienc. Salud. 2018; 16(1): $78-83$

\section{R E S U M E N}

En el presente estudio evaluamos indicadores entomológicos (índices de infección, colonización e infección natural) de ejemplares de Triatoma sordida capturados en el intra y peridomicilio de viviendas del Chaco Paraguayo en el período 2014 al 2016. El objetivo fue comparar con los hallazgos previamente reportados en el periodo 2010 al 2013 en la misma región. Además se ha incorporado la detección de fuente de alimentación como complemento para definir el riesgo de domiciliación de este vector secundario. Se aplicaron técnicas moleculares asociados a indicadores entomológicos y epidemiológicos a 220 ejemplares de $T$. sordida capturados en 67 viviendas de 24 localidades del Chaco. Se detectó infestación y colonización por $T$. sordida en el intradomicilio 13/67 (19\%) y 5/13 $(38 \%)$ y en el peridomicilio $54 / 67$ (81\%) y $43 / 54(80 \%)$, respectivamente. Se detectó infección con $T$. cruzi en un $17,3 \%$ de los ejemplares analizados. La fuente de alimentación pudo detectarse en tan solo 13 de 220 ejemplares (6\%), todos resultaron positivos para sangre de gallina y correspondían a captura en el peridomicilio. El índice de infestación intradomiciliar fue del $19 \%$, superior al de años anteriores y similar a las zonas históricamente endémicas de la Región Occidental (18\%-20\%). El riesgo de transmisión intradomiciliar sigue siendo elevado porque en 3 de 5 viviendas con ninfas de $T$. sordida, los ejemplares estaban infectados con $T$. cruzi. Los indicadores entomológicos obtenidos en el presente estudio concuerdan con resultados previos de nuestro equipo, y confirman la capacidad vectorial de este triatomino secundario en la transmisión de la enfermedad de Chagas.

Palabras clave: $T$. sordida, infestación, colonización, infección natural, fuente de alimentación.

\section{Blood meal source of specimens of Triatoma sordida in an area with high risk of domiciliation in the Paraguayan Chaco}

\section{A B S T R A C T}

In the present study we evaluated entomological indicators (infection rates, colonization and natural infection) of Triatoma sordida specimens captured in the intra and peridomicile of households of the Paraguayan Chaco in the period 2014 to 2016. The objective was to compare with the previously reported findings in the period 2010 to 2013 in the same region. In addition, the detection of the blood meal source has been incorporated as a complement to define the risk of domiciliation of this secondary vector. Molecular techniques associated with entomological and epidemiological indicators were applied to 220 
T. sordida specimens captured in 67 dwellings in 24 locations in the Chaco. Infestation and colonization by $T$. sordida was detected in the intradomicile $13 / 67(19 \%)$ and $5 / 13(38 \%)$ and in the peridomiciliary $54 / 67(81 \%)$ and $43 / 54(80 \%)$, respectively. Infection with $T$. cruzi was detected in $17.3 \%$ of the samples analyzed. The blood meal source could be detected in only 13 of 220 specimens (6\%), all were positive for chicken blood and corresponded to capture in the peridomicile. The rate of intradomiciliary infestation was $19 \%$, higher than in previous years and similar to the historically endemic areas of the Western Region (18\%-20\%). The risk of intradomiciliary transmission remains high because in 3 out of 5 dwellings with nymphs of $T$. sordida, the specimens were infected with $T$. cruzi. The entomological indicators obtained in the present study agree with previous results of our team, and confirm the vectorial capacity of this secondary triatomine in the transmission of Chagas disease.

Key words: T. sordida, infestation, colonization, natural infection, blood meal source.

\section{INTRODUCCIÓN}

La enfermedad de Chagas es causada por el parásito Trypanosoma cruzi cuyo vector principal en la Región del Cono Sur de América Latina es el Triatoma infestans. Esta vía de transmisión ha disminuido notablemente en la última década, gracias a las decisiones políticas y técnicas de los países que con actividades sostenidas de control vectorial han logrado el corte de la transmisión intradomiciliar de $T$. cruzi por esta especie ${ }^{(1,2)}$. En la actualidad existe un riesgo permanente de re-infestación de las viviendas desde focos residuales de $T$. infestans como también el riesgo de la invasión de viviendas por especies secundarias si no se llevan a cabo acciones de vigilancia entomológica sostenidas en el tiempo y espacio ${ }^{(2,3)}$. Muchas especies nativas adquieren importancia epidemiológica debido a la capacidad de intercambio entre hábitats selváticos y domésticos, así como por su potencialidad para reemplazar el nicho ecológico de triatominos estrictamente domiciliarios., Luego de la disminución de estas especies por la acciones de control y vigilancia vectorial, adquieren importancia epidemiológica las especies secundarias, entre éstas especies se destaca el $T$. sordida considerado como vector secundario en la transmisión de T. cruzi ${ }^{(2,4)}$. El gran Chaco es una eco-región que incluye Argentina, Bolivia y Paraguay donde la trasmisión vectorial de la enfermedad de Chagas por $T$. infestans (vector principal) constituye hasta la fecha un problema de salud pública. El Chaco paraguayo ocupa el $25 \%$ de esta región, caracterizada por una baja densidad poblacional y localidades dispersas. Según un estudio reciente realizado por nuestro grupo de trabajo se ha evidenciado que la especie $T$. sordida posee una capacidad adaptativa en el domicilio y se estima un $87 \%$ de riesgo de transmisión de $T$. cruzi intradomiciliar en la Región Occidental ${ }^{(2)}$. Existen otros estudios de nuestro grupo realizados sobre la capacidad vectorial de la especie $T$. sordida provenientes de áreas endémicas de la Región Oriental que reportaron en el departamento de Concepción $44 \%$ de colonización intradomiciliar y $10,6 \%$ de infección natural ${ }^{(5)}$ y en el departamento de Paraguarí $27 \%$ de colonización intradomiciliar y $12,5 \%$ de infección natural ${ }^{(6)}$. En la región del Chaco paraguayo, el control vectorial ha sido limitado por el aislamiento de las viviendas, la persistencia de la infestación doméstica, y las costumbres culturales de los habitantes (tribus indígenas). La detección de infección natural e identificación de la fuente de alimentación de triatominos permite conocer sus huéspedes e indagar sobre su comportamiento y riesgo para transmitir $T$. cruzi, razón por la cual se propuso realizar este estudio.

\section{MATERIALES Y MÉTODOS}

Área de estudio y población: Se analizaron 220 ejemplares de $T$. sordida capturados entre 2014 y 2016 en 67 viviendas de 24 localidades distribuidos en 8 distritos de los tres departamentos de la Región Occidental o Chaco. En el departamento de Pte. Hayes los ejemplares fueron capturados en 50 viviendas ubicadas en 18 localidades dispersas en 4 distritos; mientras que en Boquerón y Alto Paraguay, los ejemplares fueron capturados en 5 y 12 viviendas, situadas en 4 y 2 localidades de 2 distritos respectivamente. Fueron registrados los datos epidemiológicos como sitio de captura y estadío de los triatominos capturados en las viviendas.

Los ejemplares de $T$. sordida fueron clasificados taxonómicamente según Lent y Wygodzinsky ${ }^{(7)}$. 
Disección de triatominos: Se realizó la disección de cada uno de los 220 ejemplares de $T$. sordida para la posterior extracción de ADN, cada ejemplar disectado fue colocado en tubos que contenían buffer de lisis compuesto por soluciones de Tris $\mathrm{pH} 7,4, \mathrm{EDTA}, \mathrm{NaCl}$ y SDS.

Extracción de ADN: Se realizó la incubación de cada tubo con 5 ul de proteinasa K (20 $\mathrm{ug} / \mathrm{ml}$ ) a $60^{\circ} \mathrm{C}$ por 60 minutos. La enzima fue inactivada por calentamiento a $95^{\circ} \mathrm{C}$ por 10 minutos. EI ADN fue purificado por el método: fenol-cloroformo descrito por Sambrook et $a^{(8)}$, se realizó la precipitación con etanol, previa adición de 20 ug de Glucógeno y Acetato de sodio $3 \mathrm{M}, \mathrm{pH} 5,4$ en un $10 \%$ del volumen recuperado. El ADN se resuspendió en 100ul de agua destilada estéril.

Infección natural con T. cruzi: La identificación especie específica de $T$. cruzi fue por el método PCR empleando como blanco las secuencias repetitivas de ADN nuclear (microsatélites) de $T$. cruzi, con los cebadores TCZ1 y TCZ2 descrito por Moser et $a I^{(9)}$ y ADN kinetoplastídico con cebadores 121 y 122 descrito por Degrave et al.

Los productos amplificados fueron analizados en geles de agarosa al $2 \%$, teñidos con bromuro de etidio y visualizados bajo un transiluminador UV.

Detección de fuente de alimentación: Para la identificación de la fuente de alimento se emplearon los métodos: PCR y RFLP. Se realizó primeramente un PCR que consistió en la amplificación del gen de citocromo b $(C y t b)$, ADN mitocondrial, se utilizaron los cebadores Cyt $b 1$ y Cyt $b 2$ descrito por Osnaghi et $a l^{(10)}$.

Posteriormente se realizó la digestión de los amplificados de Cyt $b$ con 2 enzimas de restricción: Hae III y Mwo I para la determinación de los patrones de alimentación, se emplearon ADN de 4 especies como controles positivos: humano, ave (gallina), marsupial/roedor y perro según protocolo descrito por Chena et al ${ }^{(11)}$. Los productos amplificados fueron analizados en geles de agarosa al $2 \%$, teñidos con bromuro de etidio y visualizados bajo un transiluminador UV.

\section{RESULTADOS}

Se analizaron un total de 220 ejemplares de $T$. sordida colectados de 67 viviendas del Chaco Paraguayo en el período 2014-2016. La captura de los ejemplares en cada vivienda corresponde a una actividad realizada dentro de la vigilancia entomológica del SENEPA (MSP y BS), en localidades previamente fumigadas por la presencia de $T$. infestans.

En cuanto al lugar de captura y estadío, se observó que el $91 \%(201 / 220)$ de los ejemplares fueron capturados en el peridomicilio de las viviendas y el 55\% (111 de 201) eran ninfas. Tan solo 5 de las 67 viviendas infestadas (7,5\%) tenían ejemplares de $T$. sordida en el intradomicilio y en las mismas se encontraron ninfas (colonización) (Tabla 1).

Identificación de T. cruzi: Un total de 38 de 220 ejemplares analizados $(17,3 \%)$ dieron positivo por PCR para $T$. cruzi. En la Figura 1 se puede observar amplificados de ADN de $T$. cruzi con cebadores TCZ1 y TCZ2 con productos de 198pb.

Tabla 1. Distribución de las 67 viviendas infestadas con $T$. sordida según estadío y lugar de captura de los 220 ejemplares analizados.

\section{Viviendas con $T$. sordida}

\begin{tabular}{clllc}
$\begin{array}{l}\text { Ejemplares de } \\
\text { T. sordida } \\
\text { Estadío }\end{array}$ & INTRADOMICILIO & $\begin{array}{c}\text { Viviendas } \\
\text { Total } \\
\text { infestadas }\end{array}$ & PERIDOMICILIO & $\begin{array}{c}\text { Viviendas } \\
\text { Total infestadas }\end{array}$ \\
\hline NINFAS $\left(^{*}\right)$ & $10(53 \%)$ & $\begin{array}{l}5(3 \text { con } T . \\
\text { sordida } \\
\text { infectadas })\end{array}$ & $111(55 \%)$ & 43 \\
ADULTOS & $9(47 \%)$ & 8 & $90(45 \%)$ & 11 \\
Total & 19 & 13 & 201 & 54 \\
\hline \multicolumn{2}{c}{$(*)$ La presencia de ninfas en el intradomicilio de las viviendas es un indicador de colonización. }
\end{tabular}




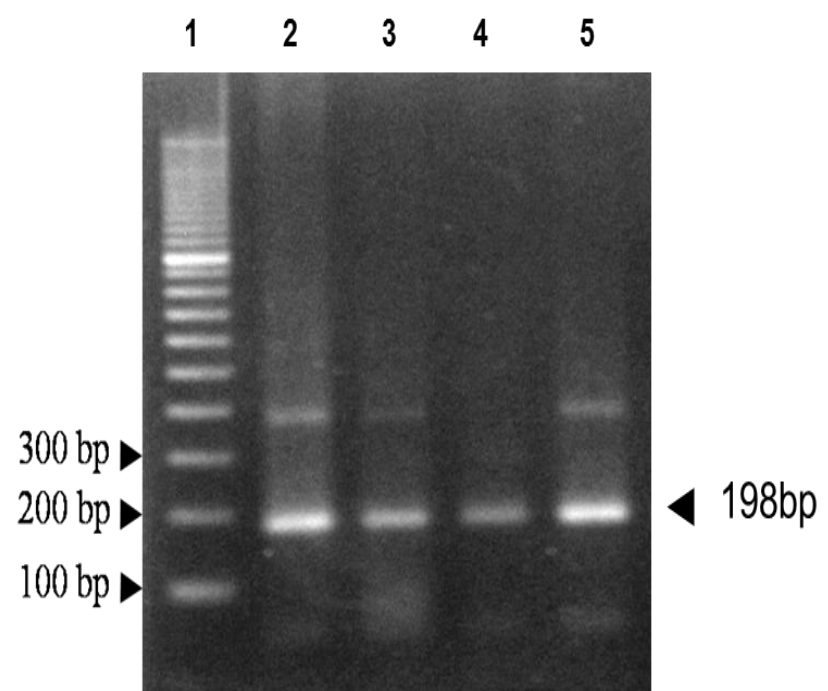

Figura 1. Amplificación de ADN de T. cruzi en T. sordida. Carril 1: Marcador de peso molecular. Carril 2: control positivo de reacción de PCR equivalente a 100 parásitos. Carril 3: control positivo de reacción equivalente a 10 parásitos. Carril 4: muestra de $T$. sordida con T. cruzi positivo correspondiente a 10 parásitos. Carril 5: muestra de T. sordida con $T$. cruzi positivo correspondiente a 100 parásitos.

Identificación de fuente de alimentación. La amplificación del gen cyt $b$ fue posible detectar en 13 ejemplares de los 220 analizados (6\%), siendo todos del peridomicilio. En la figura 2 se puede observar los amplificados del gen $c y t b$ de los ejemplares de $T$. sordida con un tamaño correspondiente a $358 \mathrm{bp}$, posteriormente éstos amplificados dieron con las 2 enzimas de restricción un patrón de sangre de gallina como fuente de alimentación. En la figura 3 se pueden observar los patrones de sangre de gallina obtenidos con la enzima Hae III con productos de 74, 125 y $159 \mathrm{bp}$.

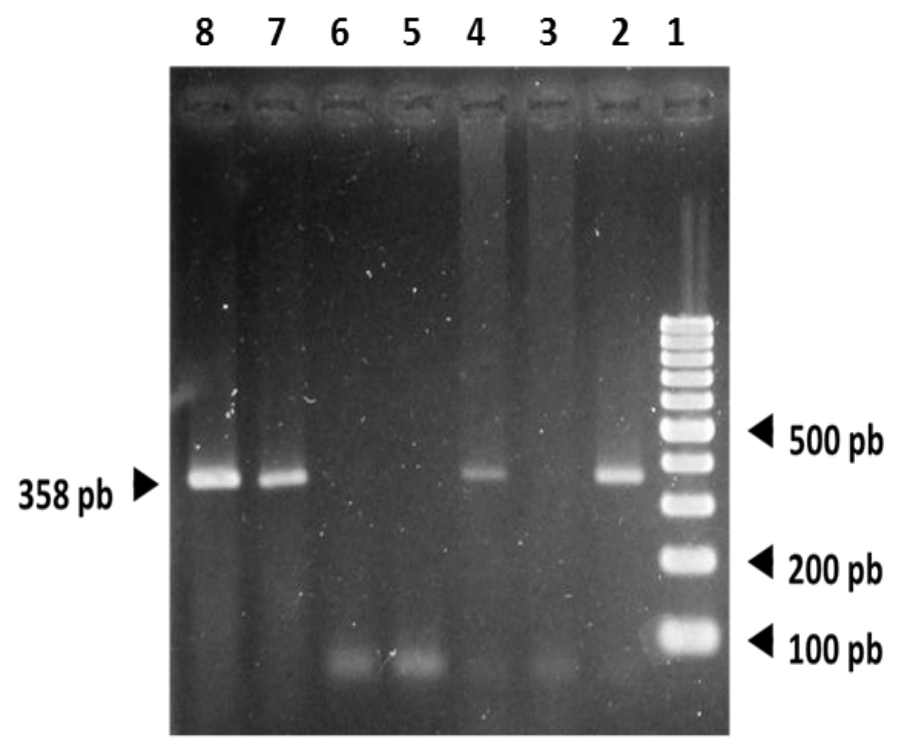

Figura 2. Amplificación del gen Cyt b en T. sordida. Carril 1: Marcador de peso molecular. Carril 2: muestra de T. sordida; cyt b positiva. Carril 3: control negativo de reacción. Carril 4: muestra de $T$. sordida positiva. Carril 5: control negativo de extracción de ADN. Carril 6: muestra de T. sordida sin producto amplificado (negativa). Carril 7: control positivo de ADN de la especie ave (gallina). Carril 8: control positivo de ADN humano. 


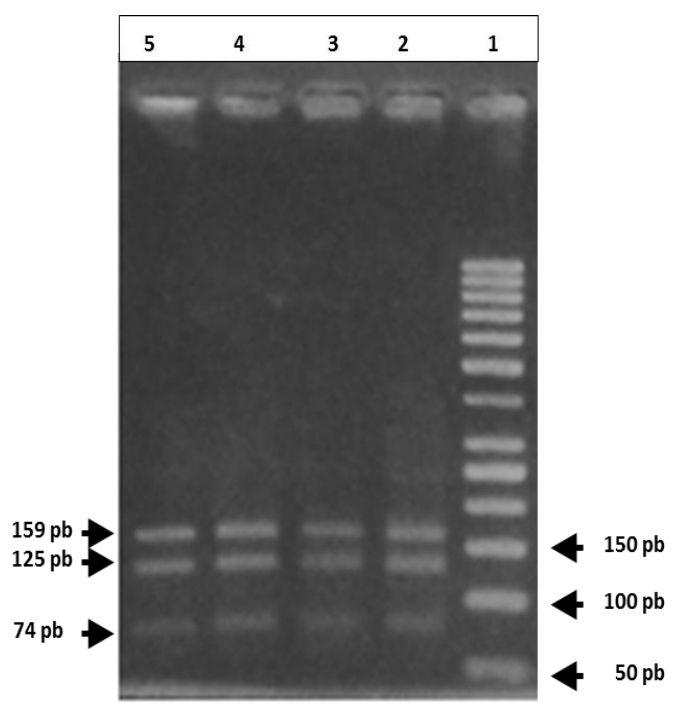

Figura 3. Digestión del gen Cyt b con la enzima de restricción Hae III. Carril1: Marcador de peso molecular. Carril 2: control positivo patrón de sangre de gallina. Carriles 3, 4, y 5: muestras de T. sordida con patrón de sangre de gallina.

\section{DISCUSIÓN}

Los índices de colonización intradomiciliar (38\%) y de infección natural con $T$. cruzi $(17,3 \%)$ detectados en este estudio son menores a lo constatado en la publicación de nuestro grupo sobre $T$. sordida del Chaco Paraguayo del 2016, donde se detectaron $52 \%$ y $21 \%$ respectivamente ${ }^{(2)}$, esto tal vez se atribuya al número de viviendas evaluadas. Sin embargo, el riesgo de transmisión intradomiciliar es elevado porque en las 5 viviendas ya se observó colonización de $T$. sordida, y en 3 de ellas se detectaron ejemplares infectados con T. cruzi, lo cual debe ser observado porque en las comunidades indígenas del Chaco la población humana infectada supera el $50 \%$ y las ninfas deben alimentarse para mudar de estadío. Por otro lado la preferencia de $T$. sordida en el peridomicilio sigue siendo mayor con respecto al domicilio, teniendo en cuenta que de las 67 viviendas infestadas 43 (64\%) presentaban como sitio de captura el peridomicilio. Así mismo, la densidad de ejemplares de $T$. sordida en el peridomicilio es mucho mayor que en el domicilio, 201 triatominos en 54 viviendas y 19 triatominos en 13 viviendas respectivamente; lo que evidencia una adaptación aún muy fuerte de $T$. sordida al peridomicilio, como ocurre en regiones de Argentina, incluyendo el Chaco Argentino ${ }^{(14,15)}$.

La especie $T$. sordida representa un problema epidemiológico en numerosos países del Cono Sur debido a su amplia valencia ecológica, que se manifiesta en su hallazgo en biotopos silvestres, peridomésticos, así como dentro de la vivienda(12-14,19). Con relación a la infección natural con $T$. cruzi, el índice detectado fue del 17,3\%, superior a lo detectado en Argentina por ejemplo, donde se había reportado desde el $2 \%$ hasta el $12 \%$ de infección natural en ejemplares de $T$. sordida capturados en el peridomicilio ${ }^{(15,16)}$.

La detección de la fuente de alimentación realizada por el método PCR-RFLPC ${ }^{(20)}$ con patrón de sangre de gallina se encontró en un $6 \%$ de los ejemplares analizados. La detección de sangre de gallina en el peridomicilio y no de roedores silvestres, reduce el riesgo de infección natural en estos ejemplares. En Brasil ya se habían reportado datos similares en cuanto a hábitos alimenticios de ésta especie de triatomino en regiones de ese país $^{(18,20)}$. En Paraguay nuestro grupo de trabajo ha realizado varios estudios con $T$. sordida en lo referente a fuente de alimentación por el método PCR-RFLP del gen cyt $b$ en ejemplares de la Región Oriental, donde por ejemplo en un estudio sobre un total de 216 ejemplares capturados en el departamento de Concepción, 11 dieron $c y t b$ positivos, de los cuales 8 dieron patrón de sangre humana y 1 ejemplar dio sangre de gallina como patrón alimenticio ${ }^{(5)}$. Otro estudio del mismo grupo reveló que en un total de 247 ejemplares de $T$. sordida procedentes del departamento de Paraguarí se detectaron 48 ejemplares cyt $b$ positivos, de los cuales 3 dieron patrón de sangre humana y 45 dieron patrón de sangre de marsupial/roedor ${ }^{(6)}$. Recientemente, en un estudio con $T$. sordida de los departamentos de Paraguarí, San Pedro y Cordillera, se detectaron como fuente de alimentación sangre humana en $11 \%, 10 \%$ y $82 \%$ respectivamente ${ }^{(17)}$. 
Los indicadores entomológicos obtenidos en el presente estudio concuerdan con resultados previos de nuestro equipo, y confirman la capacidad vectorial de este triatomino secundario en la transmisión de la enfermedad de Chagas.

\section{REFERENCIAS BIBLIOGRAFICAS}

1- Silveira AC, Rojas de Arias A, Segura E, Guillén G, Russomando G, Schenone $H$, et al. El control de la Enfermedad de Chagas en los países del Cono Sur de América. Historia de una Iniciativa Internacional. 1991/2001. OPAS, Universidade Federal do Triângulo Mineiro, Uberaba; 2002. 316.

2- Sánchez Z, Russomando G, Pineda D, Guillén L, Paredes B, Villalba de Feltes C. Triatoma sordida: indicadores de adaptación y transmisión de Trypanosoma cruzi en intradomicilio del Chaco Paraguayo. Mem Inst Investig Cienc Salud 2016; 14(3):96101.

3- Noireau F, Gutierrez T, Flores R. Brenier F, Bosseno MF, Wisnivesky- Colli C. Ecogenetics of $T$. sordida and T. guayasana (Hemiptera: Reduviidae) in the Bolivian Chaco. Mem Inst Oswaldo Cruz 1999; 94(4): 451-57.

4- OPS Organización Panamericana de la Salud. Taller del Cono Sur enfermedad de Chagas. Conceptualización de la vigilancia epidemiológica. Bs. As. Argentina. 2003.

5- Noireau F, Flores R, Gutierrez T, Abad-Franch F, Flores E, Vargas F. Natural ecotopes of Triatoma infestans dark morph and other sylvatic triatomines in the Bolivian Chaco. Trans R Soc Trop Med Hyg 2000; 94: 23-7.

6- Sánchez León Z, Russomando G, Guillén R. Enfermedad de Chagas: estudio de un vector secundario. Editorial académica española. 2012.

7- Sánchez Z, Chena L, Nara E, Espinola EE, Ferreira E, Paredes B. et al. Alta frecuencia de sangre humana en Triatoma sordida (vector secundario de Chagas), infección natural con Trypanosoma cruzi y riesgo de domiciliación en viviendas rurales del departamento Paraguarí. IX Congreso Paraguayo de Infectología. AsunciónParaguay. 2013.

8- Lent H, Wygodzinsky P. Revision of Triatominae (Hemiptera: Reduviidae), and the other significanse as vectors of Chagas disease. Bull Am Mus Nat Hist 1979; 163: 123-27.

9- Sambrook J, Fritsch E, Maniatis T. Molecular Cloning. A Laboratory Manual. Cold Spring Harbor Laboratory Press. New York E.U.A 1994.

10-Moser DR, Kirchhoff LV, Donelson JE. Detection of Trypanosoma cruzi by DNA amplification using the polymerase chain reaction. J Clin Microbiol 1989; (7): 1477-82.

11-Osnaghi MA, Chavshin AR, Vatandoost $H$. Analysis of mosquito bloodmeals sing RFLP markers. Exp. Parasitol. 2006; 114(4): 259.64

12-Chena L, Nara E, Sánchez Z, Espinola E, Russomando G. Estandarización de la técnica
PCR-RFLP del gen mitocondrial cyt $b$ como herramienta para la identificación de fuentes de alimentación de insectos hematófagos. Mem. Inst. Investig. Cienc. Salud, Vol 12 (2). $2014 ; 33.42$

13- Noireau F, Breniere F, Ordoñez J, Cardozo L,Morochi W, Gutierrez T, Bosseno MF, García S, Vargas F, Yaksic N, Dujardin JP, Peredo C, Wisnivesky-Colli C. Low probability of transmission of Trypanosoma cruzi to humans by domiciliary Triatoma sordida in Bolivia. Trans. R. Soc. Trop. Med. Hyg. 1997; 91, 653-6.

14- Breniere SF, Morochi W, Bosseno MF, Ordoñez J, Gutierrez T, Vargas F, et al. Trypanosoma with domestic Triatoma sordida in Bolivia. Acta Tropica 71.1998; 269-83.

15- Macchiaverna NP, Gaspe MS, Enriquez GF, Tomassone L, Gurtler RE, Cardinal MV. Trypanosoma cruzi infection in Triatoma sordida before and after community-wide residual insecticide spraying in the Argentina an Chaco. Acta Tropica 2015; 143, 97-102.

16- Oscherov EB, Bar ME, Damborsky MP, Milano A, Avalos G, Borda M. Epidemiología de la enfermedad de Chagas, Departamento General Paz, Argentina. Rev Saúde Pública 2003; 37(1):59-64.

17- Bar ME, Wisnivesky-Colli C. Triatoma sordida Stal 1859 (Hemiptera, Reduviidae: Triatominae) in palms of northeastern Argentina. Mem Inst Oswaldo Cruz 2001;(96): 895-9.

18- Sánchez Z, Pineda D, Guillén L, Paredes B, Russomando G. Triatoma sordida en viviendas de áreas rurales del Paraguay: Infección natural con Trypanosoma cruzi y Fuente de alimentación. XI Congreso Paraguayo de Infectología. AsunciónParaguay 2017.

19- Forattini OP, Barata JMS, Santos JLF, Silvera AC. Hábitos alimentares: infeccao natural e distribuicao de triatomíneos domiciliados na regiao central do Brasil. Rev Saúde Públ $1982 ; 16,171-204$.

20- González-Britez N, Vega C, Rolón M, Rojas de Arias A. Triatomines and other arthopods in bird nests in native and Mennonite communities of the Paraguayan Chaco. Abstracts of XV Internacional Congress for Tropical Medicine and Malaria, CartagenaColombia 2000; (2): 89.

21-Mota J, Chacon JC, Gutierrez AF, Sánchez V, Wirtz $A$, Ordoñez $R$ et al. Identification of blood meal source and Infection with Trypanosoma cruzi of Chagas Disease Vectors using a Multiplex Cytocrhrome b Polimerase Chain Reaction, Vector Borne and Zoonotic Diseases. 2007; 7(4): 617-27. 